\title{
The impact of breast cancer treatments on sleep quality 1 year after cancer diagnosis
}

\author{
Filipa Fontes $^{1}$ - Susana Pereira ${ }^{1,2}$ - Ana Rute $\operatorname{Costa}^{1} \cdot$ Marta Gonçalves $^{1,3}$. \\ Nuno Lunet ${ }^{1,4}$
}

Received: 11 April 2016 / Accepted: 31 May 2017 / Published online: 16 June 2017

(C) Springer-Verlag GmbH Germany 2017

\begin{abstract}
Purpose The increasing number of women living longer with potential side effects of breast cancer treatment highlights the need of a comprehensive assessment of its burden. Therefore, we aimed to quantify the relation between different breast cancer treatments and sleep quality 1 year after diagnosis. Methods A cohort of 502 newly diagnosed breast cancer patients was prospectively followed. Sleep quality was evaluated with the Pittsburgh Sleep Quality Index (PSQI), at baseline and at the 1-year follow-up. Odds ratios (OR) were computed to quantify the association between patient characteristics and poor sleep quality (PSQI score $>5$ ) at baseline, and relative risks (RR) were computed for the association between treatments and the occurrence of poor sleep quality at 1 year.

Results A total of $60.2 \%$ of the patients had poor sleep quality before breast cancer treatments, especially those with anxiety $[\mathrm{OR}=2.86,95 \%$ confidence interval $(95 \% \mathrm{CI}) 1.92$ to 4.27$]$ or depression ( $\mathrm{OR}=5.25,95 \% \mathrm{CI} 2.01$ to 13.67). Radiotherapy increased the risk of poor sleep quality at 1 year $(\mathrm{RR}=3.71$, $95 \%$ CI 1.15 to 11.96 , for a cumulative dose $>50 \mathrm{~Gy}$ ) and there was a tendency for a higher risk in those submitted to chemotherapy, although not statistically significant.
\end{abstract}

Nuno Lunet

nlunet@med.up.pt

1 ISPUP-EPIUnit, University of Porto, Rua das Taipas, 135, 4050-600 Porto, Portugal

2 Portuguese Institute of Oncology of Porto, Rua Dr. António Bernardino de Almeida, 4200-075 Porto, Portugal

3 Sleep Medicine Center - CUF Porto Hospital, Porto, Portugal

4 Department of Clinical Epidemiology, Predictive Medicine and Public Health, Faculty of Medicine, University of Porto, Al. Prof. Hernâni Monteiro, 4200-319 Porto, Portugal
Conclusions Our study shows that sleep disturbances are frequent before cancer treatment and confirms their cooccurrence with other medical conditions, such as anxiety and depression. Different breast cancer treatments increase the risk of impaired sleep quality, therefore contributing to the global disability associated with cancer treatments.

Keywords Antineoplastic protocols · Breast cancer · Quality of life $\cdot$ Radiotherapy $\cdot$ Sleep

\section{Introduction}

Breast cancer is the second most common cancer in the world and the most frequent among women, with an estimated 1.7 million new cancer cases diagnosed in 2012 [1]. During the last few decades, survival has improved as a result of earlier diagnoses and the use of more effective and aggressive treatments [2], surpassing $80 \%$ in most developed settings [3]. The growing number of women living for longer periods with potential sequelae of breast cancer treatment makes it the worldwide leading cause of years lived with disability due to oncological diseases in women [4], which highlights the need of a comprehensive assessment of the burden associated with breast cancer treatment among survivors.

Poor sleep quality is an important contributor to low healthrelated quality of life [5, 6] and is estimated to affect up to $70 \%$ of breast cancer patients [7], depending on the method of sleep assessment and study design used. Although a high proportion of breast cancer patients may present sleep disturbances before beginning treatment $[8,9]$, cancer treatment has been associated with the occurrence of sleep disturbances among women with breast cancer $[10,11]$. In a recent systematic review, women submitted to chemotherapy (CTX), as well as radiotherapy, tended to report higher levels of sleep 
disturbances; inconsistent findings were observed regarding the different options for surgery and the use of hormonal treatment [12]. The only prospective study included in this review evaluated patients before surgery and followed them for 6 months, concluding that adjuvant CTX was associated with gradual increases in sleep disturbances that peaked at the third month and then decreased until 6 months after surgery [13].

Although previous studies have addressed the relation between breast cancer treatments and sleep disturbances, the achievement of more robust findings was hampered by limitations such as the absence of control for confounding, the use of non-standardized measures to evaluate sleep quality and the scarcity of longitudinal analyses taking into account sleep quality of the patients at baseline, in addition to some lack of detail in the description of all treatments [12]. Data from prospective studies with an evaluation of participants prior to treatments and providing a detailed assessment of the effect of breast cancer treatments may contribute to a more accurate characterization of sleep quality determinants. Therefore, we aimed to quantify the relation between different breast cancer treatments and sleep quality 1 year after breast cancer diagnosis, using a contemporary prospective cohort of women in the early stages of the disease, and with the baseline evaluation performed before treatments.

\section{Methods}

We conducted a prospective study with newly diagnosed breast cancer women, followed for 1 year, as described in detail elsewhere [14].

\section{Patients and settings}

Participants were consecutively recruited in 2012, among patients with a breast cancer diagnosed within the previous 3 months and proposed for surgery, aged 18 years or older, and admitted to the Breast Clinic of the Portuguese Institute of Oncology of Porto. We excluded those previously treated with CTX and/or radiotherapy in the chest or axillary areas for other primary cancers, those that had received any treatment for breast cancer before, and those with a high probability of cognitive impairment (a score lower than 17, or lower than 16 for women over 65 years, in the Montreal Cognitive Assessment) [15].

The cohort included 506 patients with incident breast cancer, from whom 502 (99.2\%) completed the 1-year follow-up evaluation without missing data in the outcome studied (one patient died, two patients abandoned the study, and one patient had missing information on sleep quality at 1 year) and were therefore included in the present analysis.

\section{Data collection}

For the analysis, data were collected in two distinct moments: at baseline (after diagnosis but before any cancer treatment) and 1 year after enrollment. The median time [percentile 25, percentile 75 (P25, P75)] between diagnosis (date of the first histological confirmation) and the baseline evaluation was 25 days (18-32).

At baseline, sociodemographic data were collected using a structured questionnaire and clinical records were reviewed for cancer stage and menopausal status. Cancer stage was classified according to the American Joint Committee on Cancer Staging Manual [16]. When menopausal status was not specified, all women older than 60 , women who underwent a bilateral ovariectomy, and those with an intact uterus and amenorrheic for at least 1 year prior to the breast cancer diagnosis were classified as postmenopausal, otherwise as premenopausal [17]. For analysis, age was categorized, using the mean of the distribution among all participants as the cutoff ( $\leq 55$ and $>55$ years). Education was collected as a continuous variable considering the complete years of schooling and later categorized as 4 years or less (primary education), 5 to 9 years (lower secondary school), and 10 years or more (upper secondary and post-secondary education).

At the 1-year follow-up, clinical records were reviewed for cancer treatments performed during the previous year.

The Hospital Anxiety and Depression Scale (HADS) was used to evaluate anxiety and depression at baseline [18, 19]. Scores for each subscale range from 0 to 21 , and a score greater than or equal to 11 for each was considered to be indicative of clinically significant anxiety and/or depression, as applicable $[18,20]$.

Sleep quality at baseline and at the 1-year follow-up evaluation was assessed using the Pittsburgh Sleep Quality Index (PSQI) [21, 22]. The PSQI consists of 19 questions measuring seven components, including subjective sleep quality, sleep latency, sleep duration, habitual sleep efficiency, sleep disturbances, use of sleep medication, and daytime dysfunction. Items are summed to provide a global sleep quality score ranging from 0 to 21 , with higher scores indicating worse sleep quality; a score greater than 5 indicates poor sleep quality [21].

\section{Statistical analysis}

Crude and adjusted odds ratios (OR), and corresponding 95\% confidence intervals $(95 \% \mathrm{CI})$, for the relation between sociodemographic and clinical characteristics of the patients, and the presence of poor sleep quality at baseline were computed using logistic regression. Relative risks (RR) and 95\%CI were computed using the Poisson regression, taking into account the time between the baseline and the 1-year follow-up evaluation, to identify predictors of improvement $(\mathrm{PSQI} \leq 5)$ or deterioration (PSQI $>5$ ) of sleep quality, in those 
with poor and good sleep quality at baseline, respectively. Variables included in each model are those considered, as a result of previous studies or expert knowledge, to be associated with both the exposure and the outcome, but influenced by neither, and are described in the footnotes of the corresponding table or figure. Whenever overlapping treatments were considered in the multivariable models, each treatment was included as an independent dichotomous variable.

Sample characteristics are presented as counts and proportions for categorical variables, mean and standard deviation (SD) for quantitative variables with an approximately symmetrical distribution, and median (P25-P75) for quantitative variables with a markedly asymmetrical distribution. Statistical analyses were conducted using STATA $®$, version 11.2 (StataCorp, College Station, TX, USA).

\section{Results}

\section{Patients' characteristics}

The main sociodemographic and clinical characteristics of the patients are presented in Table 1. At baseline, $47.2 \%$ had more than 55 years of age and $71.5 \%$ had less than 10 years of education. Most women were diagnosed with cancer stage 0 or I (53.6\%). Few participants (3.8\%) did not undergo adjuvant/ neoadjuvant therapy with $14.9 \%$ performing only one type. Among the latter, 0.6, 1.4, and $12.9 \%$ performed chemotherapy, radiotherapy, and endocrine therapy, respectively. A total of $81.3 \%$ of the participants underwent at least two types of treatments; the most common combinations were radiotherapy and endocrine therapy (62.0\%), chemotherapy and endocrine therapy (49.8\%), and chemotherapy and radiotherapy (49.6\%).

The mean (SD) time between the baseline and the 1-year follow-up evaluation was 379.0 days (28.6). Among those performing surgery, chemotherapy, radiotherapy, or brachytherapy, the 1-year follow-up evaluation occurred 337.2 (58.5), 205.7 (44.5), 164.8 (73.2), and 195.0 days (82.7), respectively, after the end of the treatment. Those performing chemotherapy and radiotherapy were under treatment for a median (P25-P75) of 105 days (103-113) and 34 days (32-42), respectively.

\section{Factors associated with poor sleep quality at baseline}

At baseline, the median (P25, P75) PSQI score was $7(4,10)$, with $60.2 \%$ of the patients with poor sleep quality before breast cancer treatment (PSQI score $>5$ ). A total of $59.8 \%$ reported sleep-onset latency or wakefulness after a sleep onset of more than 30 min with a frequency of at least three times a week.

The association between different patient characteristics and the presence of poor sleep quality at baseline is reported in Table 2. Anxiety and depression were associated with an odds of poor sleep quality approximately three and fivefold higher, respectively. No statistically significant associations were observed for age, education, menopausal status, and cancer stage.

\section{Sleep quality during the first year after diagnosis}

The median (P25, P75) PSQI score variation between the baseline and the 1-year follow-up evaluation was $0(-2,2)$. However, $47.2 \%$ patients got worse (an increase in the PSQI score by at least one point), especially those with good sleep quality prior to treatment (58.5\% with good sleep quality versus $39.7 \%$ with poor sleep quality, $P \leq 0.001$ ) and $39.6 \%$ showed an improvement in their sleep quality, especially those with poor sleep quality at baseline $(20.5 \%$ with good sleep quality versus $52.3 \%$ with poor sleep quality, $P \leq 0.001$ ). A total of 31.9 and $39.4 \%$ of the participants reported using sleep medication at least three times per week in the preceding month, at baseline and at the 1-year follow-up, respectively.

\section{Predictors of the variation of sleep quality}

Figure 1 depicts the adjusted RR for the relation between different patient characteristics and the occurrence of poor sleep quality (PSQI $>5$ ) at 1 year, among those with good sleep quality at baseline. Radiotherapy was significantly associated with a higher risk of poor sleep quality ( $P$ value for trend $=0.032$ ) and there was a tendency for a higher risk among those submitted to mastectomy and those that underwent CTX, although differences were not statistically significant.

Figure 2 depicts the adjusted RR for the relation between different patient characteristics and the occurrence of good sleep quality (PSQI $\leq 5)$ at 1 year, among those with poor sleep quality at baseline. There was a tendency for a higher risk of good sleep quality in more educated women $(P$ value for trend $=0.008$ ) and mastectomy was associated with a lower risk of good sleep quality, though not statistically significant.

\section{Discussion}

This study shows a high prevalence of poor sleep quality before breast cancer treatments and its strong association with anxiety and depression. Among patients with poor sleep quality at baseline, education was positively associated with an improvement in sleep quality. Among patients with good sleep quality at baseline, radiotherapy increased the risk of poor sleep quality during the follow-up.

Although direct comparisons are difficult due to the heterogeneous characteristics of the patients evaluated and the use of different methods to assess sleep quality and/or to define the outcomes, our study yielded a prevalence of poor sleep quality prior to treatments approximately twice as high as previous estimates of insomnia [8, 9]. In a study from Ireland [8], $25.5 \%$ of the women awaiting breast surgery as a first-line 
Table 1 Sociodemographic and clinical characteristics of the patients $(N=502)$

\begin{tabular}{|c|c|c|}
\hline & & {$[N(\%)]$} \\
\hline \multirow[t]{2}{*}{ Age, years } & $\leq 55$ & $265(52.8)$ \\
\hline & $>55$ & $237(47.2)$ \\
\hline \multirow[t]{3}{*}{ Education, years } & $\leq 4$ & $216(43.0)$ \\
\hline & $5-9$ & $143(28.5)$ \\
\hline & $\geq 10$ & $143(28.5)$ \\
\hline Anxiety $^{\mathrm{a}}$ & & $190(37.9)$ \\
\hline Depression $^{\mathrm{b}}$ & & $41(8.2)$ \\
\hline Poor sleep quality ${ }^{\mathrm{c}}$ & & $302(60.2)$ \\
\hline \multirow[t]{2}{*}{ Menopausal status ${ }^{\mathrm{d}}$} & Premenopausal & $208(43.2)$ \\
\hline & Postmenopausal & $273(56.8)$ \\
\hline \multirow[t]{5}{*}{ Cancer stage } & 0 & $34(6.8)$ \\
\hline & I & $235(46.8)$ \\
\hline & II & $155(30.9)$ \\
\hline & III & 75 (14.9) \\
\hline & IV & $3(0.6)$ \\
\hline \multicolumn{3}{|l|}{ Surgery $^{\mathrm{e}}$} \\
\hline \multirow[t]{3}{*}{ Breast surgery } & Breast-conserving & 249 (49.6) \\
\hline & Mastectomy & $252(50.2)$ \\
\hline & Only axillary surgery & $1(0.2)$ \\
\hline \multirow[t]{2}{*}{ Axillary surgery } & SLNB & $315(64.7)$ \\
\hline & ALND & $172(35.3)$ \\
\hline CTX & & $298(59.4)$ \\
\hline \multirow[t]{2}{*}{ Timing } & Neoadjuvant & $34(11.4)$ \\
\hline & Adjuvant & $264(88.6)$ \\
\hline \multirow[t]{6}{*}{ CTX schemes } & Doxorubicin + cyclophosphamide $( \pm$ docetaxel $)$ & $92(30.9)$ \\
\hline & Doxorubicin + cyclophosphamide + paclitaxel & $1(0.3)$ \\
\hline & Cyclophosphamide + docetaxel & $2(0.7)$ \\
\hline & Carboplatin + docetaxel & $1(0.3)$ \\
\hline & 5-FU + epirubicin + cyclophosphamide $( \pm$ docetaxel $)$ & $201(67.4)$ \\
\hline & 5-FU + cyclophosphamide + methotrexate & $1(0.3)$ \\
\hline \multirow[t]{4}{*}{ Other adjuvant treatments } & Radiotherapy & $366(72.9)$ \\
\hline & Brachytherapy & $95(18.9)$ \\
\hline & Endocrine therapy ${ }^{\mathrm{f}}$ & $421(83.9)$ \\
\hline & Immunotherapy ${ }^{\mathrm{f}}$ & $68(13.6)$ \\
\hline
\end{tabular}

ALND axillary lymph node dissection, CTX chemotherapy, SLND sentinel lymph node dissection, 5-FU 5fluorouracil

${ }^{a}$ Defined as a score greater than or equal to 11 in the anxiety subscale of the Hospital Anxiety and Depression Scale; $N=501$ due to missing data

${ }^{\mathrm{b}}$ Defined as a score greater than or equal to 11 in the depression subscale of the Hospital Anxiety and Depression Scale

${ }^{\mathrm{c}}$ Defined as a score greater than 5 in the Pittsburgh Sleep Quality Index

${ }^{\mathrm{d}} N=481$ due to missing data

${ }^{\mathrm{e}}$ Patients who had both mastectomy and breast-conserving surgery are reported as mastectomy and patients who had both ALND and SLNB are reported as ALND

${ }^{\mathrm{f}}$ All patients began this treatment during the first year of follow-up and remained under treatment at the 1-year follow-up evaluation treatment presented insomnia (evaluated using the Insomnia Severity Index). In Canada [9], a study conducted among cancer patients during the perioperative phase, in which some women had already been submitted to surgery, showed that $36.0 \%$ of those with breast cancer had insomnia syndrome (assessed using an adaptation of the Insomnia Interview Schedule). 
Table 2 Association between sociodemographic and clinical characteristics of the patients and poor sleep quality at baseline $(N=502)$

\begin{tabular}{|c|c|c|c|}
\hline & $\begin{array}{l}\text { Patients with poor sleep quality } \\
\text { at baseline }[N(\%)]\end{array}$ & Crude OR $(95 \% \mathrm{CI})$ & Adjusted OR (95\%CI) \\
\hline \multicolumn{4}{|l|}{ Age (years) } \\
\hline$\leq 55$ & $154(58.1)$ & 1 (ref.) & \\
\hline$>55$ & $148(62.4)$ & $1.20(0.84$ to 1.72$)$ & \\
\hline \multicolumn{4}{|l|}{ Education (years) } \\
\hline$\leq 4$ & $130(60.2)$ & 1 (ref.) & 1 (ref.) \\
\hline $5-9$ & $92(64.3)$ & $1.19(0.77$ to 1.85$)$ & $1.31(0.82 \text { to } 2.10)^{\mathrm{b}}$ \\
\hline$\geq 10$ & $80(55.9)$ & $0.84(0.55$ to 1.29$)$ & $0.93(0.58 \text { to } 1.48)^{\mathrm{b}}$ \\
\hline \multicolumn{4}{|l|}{ Anxiety at baseline $\mathrm{c}^{\mathrm{c}}$} \\
\hline No & $160(51.4)$ & 1 (ref.) & 1 (ref.) \\
\hline Yes & $142(74.7)$ & $2.79(1.88$ to 4.15$)$ & $2.86(1.92 \text { to } 4.27)^{\mathrm{d}}$ \\
\hline \multicolumn{4}{|c|}{ Depression at baseline $\mathrm{e}^{\mathrm{e}}$} \\
\hline No & $266(57.7)$ & 1 (ref.) & 1 (ref.) \\
\hline Yes & $36(87.8)$ & $5.28(2.03$ to 13.70$)$ & $5.25(2.01 \text { to } 13.67)^{\mathrm{d}}$ \\
\hline \multicolumn{4}{|c|}{ Menopausal status at baseline $\mathrm{f}^{\mathrm{f}}$} \\
\hline Premenopausal & $121(58.2)$ & 1 (ref.) & 1 (ref.) \\
\hline Postmenopausal & $171(62.6)$ & $1.21(0.83$ to 1.74$)$ & $1.19(0.62 \text { to } 2.27)^{\mathrm{b}}$ \\
\hline \multicolumn{4}{|l|}{ Cancer stage } \\
\hline $0 / \mathrm{I}$ & $169(62.8)$ & 1 (ref.) & 1 (ref.) \\
\hline II & $85(54.8)$ & $0.72(0.48$ to 1.07$)$ & $0.71(0.47 \text { to } 1.06)^{\mathrm{g}}$ \\
\hline III/IV & $48(61.5)$ & $0.95(0.56$ to 1.59$)$ & $0.94(0.56 \text { to } 1.60)^{\mathrm{g}}$ \\
\hline \multicolumn{4}{|c|}{ OR odds ratio, $95 \%$ CI $95 \%$ confidence interval } \\
\hline \multicolumn{4}{|c|}{${ }^{\text {a }}$ Defined as a score greater than 5 in the Pittsburgh Sleep Quality Index } \\
\hline \multicolumn{4}{|c|}{${ }^{\mathrm{b}}$ Adjusted for age } \\
\hline \multicolumn{4}{|c|}{$\begin{array}{l}{ }^{\mathrm{c}} \text { Defined as a score greater than or equal to } 11 \text { in the anxiety subscale of the Hospital Anxiety and Depression } \\
\text { Scale; } N=501 \text { due to missing data }\end{array}$} \\
\hline \multicolumn{4}{|c|}{ d Adjusted for age, education, and cancer stage } \\
\hline \multirow{2}{*}{\multicolumn{4}{|c|}{$\begin{array}{l}\text { e Defined as a score greater than or equal to } 11 \text { in the depression subscale of the Hospital Anxiety and Depression } \\
\text { Scale } \\
{ }^{\mathrm{f}} N=481 \text { due to missing data }\end{array}$}} \\
\hline & & & \\
\hline${ }^{\mathrm{g}}$ Adjusted for age an & & & \\
\hline
\end{tabular}

Our findings of greater odds of poor sleep quality at baseline among those with anxiety and depression are consistent with the literature showing that anxiety and depression are associated with the presence of sleep disturbances in the general population [23-25] and in breast cancer patients [26, 27]. In fact, insomnia symptoms are one of the diagnostic criteria for several mood and anxiety disorders [28]. Conversely, the presence of a psychiatric disorder (e.g., depressive or anxiety) has been described as a predisposing factor for the development of sleep problems [29]. However, though it was not surprising to find an association between these symptoms, our cross-sectional data analysis precludes the assumption of the direction of an association between anxiety or depression and poor sleep quality prior to treatments.

Although fatigue was not evaluated in the present study, it may have played an important role in the prevalence and in the occurrence of poor sleep quality during the first year after cancer diagnosis. In fact, a growing number of recent studies support that fatigue may co-occur with depression and sleep disturbances as part of a symptom cluster in breast cancer patients [30-33]. Berger et al. examined patterns of circadian rhythms and their relationship with fatigue, anxiety, and depression and concluded that disrupted patterns of circadian rhythms were associated with distressing fatigue and depressive symptoms during chemotherapy [30]. Ho et al. investigated the co-occurrence of and interrelations between the three symptoms prior to, after and 6 to 8 months following adjuvant chemotherapy, and their findings revealed moderate to large bivariate associations between symptoms, prior to and following treatment [31]. Bower et al. examined the impact of fatigue on various components of health-related quality of life in a sample of breast cancer survivors 1 to 5 years after initial cancer diagnosis and concluded that more severe fatigue was associated with significantly higher levels of depression and sleep disturbance [32]. Finally, through a systematic review, Brown et al. confirm the association of fatigue with depression 
Fig. 1 Association between sociodemographic and clinical characteristics of the patients and the occurrence of poor sleep quality at 1 year, among those with good sleep quality at baseline $(N=200)$. ALND axillary lymph node dissection, $C T X$ chemotherapy, $S L N B$ sentinel lymph node dissection, 5-FU 5fluorouracil. ${ }^{\mathrm{a} C o m p u t e d}$ among those with good sleep quality at baseline (score equal or lower than 5 in the Pittsburgh Sleep Quality Index). ${ }^{\mathrm{b}}$ Defined as a score greater than or equal to 11 in the anxiety subscale of the Hospital Anxiety and Depression Scale. ${ }^{\mathrm{c}}$ Defined as a score greater than or equal to 11 in the depression subscale of the Scale. ${ }^{\mathrm{d}}$ Adjusted for age.

${ }^{\mathrm{e}}$ Adjusted for age, education, and cancer stage. ${ }^{\mathrm{f}}$ Adjusted for age and education. ${ }^{\mathrm{g}}$ Adjusted for age, education, cancer stage, and breast and axillary surgery.

${ }^{\mathrm{h}}$ Adjusted for age, education, cancer stage, and menopausal status Hospital Anxiety and Depression

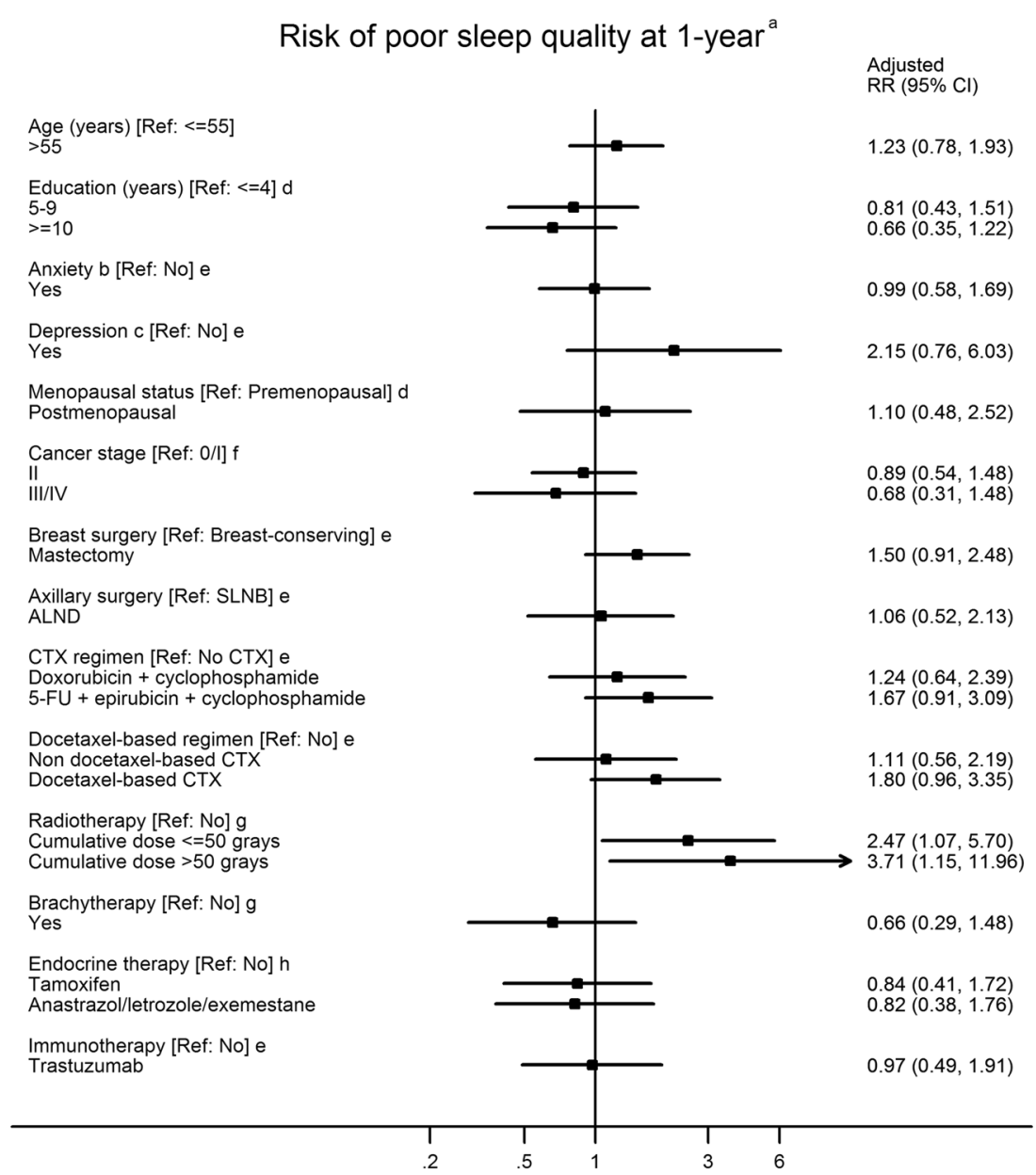

Risk of poor sleep quality at 1 -year ${ }^{a}$

and anxiety but emphasize the need of longitudinal studies to better delineate its directionality [33].

Previous results described a tendency for higher levels of sleep disturbance in women submitted to radiotherapy when compared to those not submitted to this type of treatment, though results were not statistically significant $[26,27,34]$. We found a nearly fourfold higher risk of poor sleep quality among women submitted to the highest doses of radiotherapy; the impact of physical side effects of radiation, such as skin reactions and pain, which have been demonstrated to increase with the dose administered [35], could be precipitating factors for sleep disturbances in those with good sleep quality prior to treatment.

Although not statistically significant, we found a tendency for higher levels of poor sleep quality among women submitted to CTX, which is in accordance with previous results [13, $36,37]$; the daily life disruption related with the psychological (e.g., worries, anxiety, and depression related with anticipation of side effects) and physical (e.g., hot flashes, fatigue, nausea, and vomiting) side effects of treatment could, at least in part, explain these results $[29,38]$.

The relationship between education and sleep disturbance in breast cancer patients has not been consistent across studies $[34,39,40]$. Nevertheless, our findings may reflect the fact that more educated women may have better knowledge regarding breast cancer prognosis and treatment-related disability that can contribute to minimize the physical and psychological impact of cancer diagnosis and cancer treatment on sleep quality.

The present study provides a comprehensive assessment of the impact of breast cancer treatment on sleep quality. Its major strengths are the longitudinal analysis of sleep quality, with an evaluation prior to treatments, the nearly complete follow-up of the cohort, the use of standardized sleepspecific measures, and the use of HADS to evaluate anxiety and depression. The fact that HADS does not specifically take into account symptoms such as loss of appetite, fatigue, and sleep disturbance, which may result from either depression or physical illness, is one of the major strengths of this instrument, therefore preventing false positives due to underlying medical conditions [18]. Since sleep disturbances are not directly contributing to the HADS score, this results in a more meaningful assessment of the association between sleep quality and depression in our analysis. However, some limitations need to be addressed. Our study was based on a cohort of a large number of participants, which precluded the use of objective measures of sleep quality (actigraphy or 
Fig. 2 Association between sociodemographic and clinical characteristics of the patients and the occurrence of good sleep quality at 1 year, among those with poor sleep quality at baseline $(N=302)$. ALND axillary lymph node dissection, $C T X$ chemotherapy, $S L N B$ sentinel lymph node dissection, 5-FU 5fluorouracil. ${ }^{\mathrm{a} C}$ Computed among those with poor sleep quality at baseline (score greater than 5 in the Pittsburgh Sleep Quality Index). ${ }^{\mathrm{b}}$ Anxiety defined as a score greater than or equal to 11 in the anxiety subscale of the Hospital Anxiety and Depression Scale. ${ }^{c}$ Depression defined as a score greater than or equal to 11 in the depression subscale of the Hospital Anxiety and Depression Scale. ${ }^{\mathrm{d}}$ Adjusted for age.

${ }^{\mathrm{e}}$ Adjusted for age, education, and cancer stage. ${ }^{\mathrm{f}}$ Adjusted for age and education. ${ }^{\mathrm{g}}$ Adjusted for age, education, cancer stage, and breast and axillary surgery.

${ }^{\mathrm{h}}$ Adjusted for age, education, cancer stage, and menopausal status

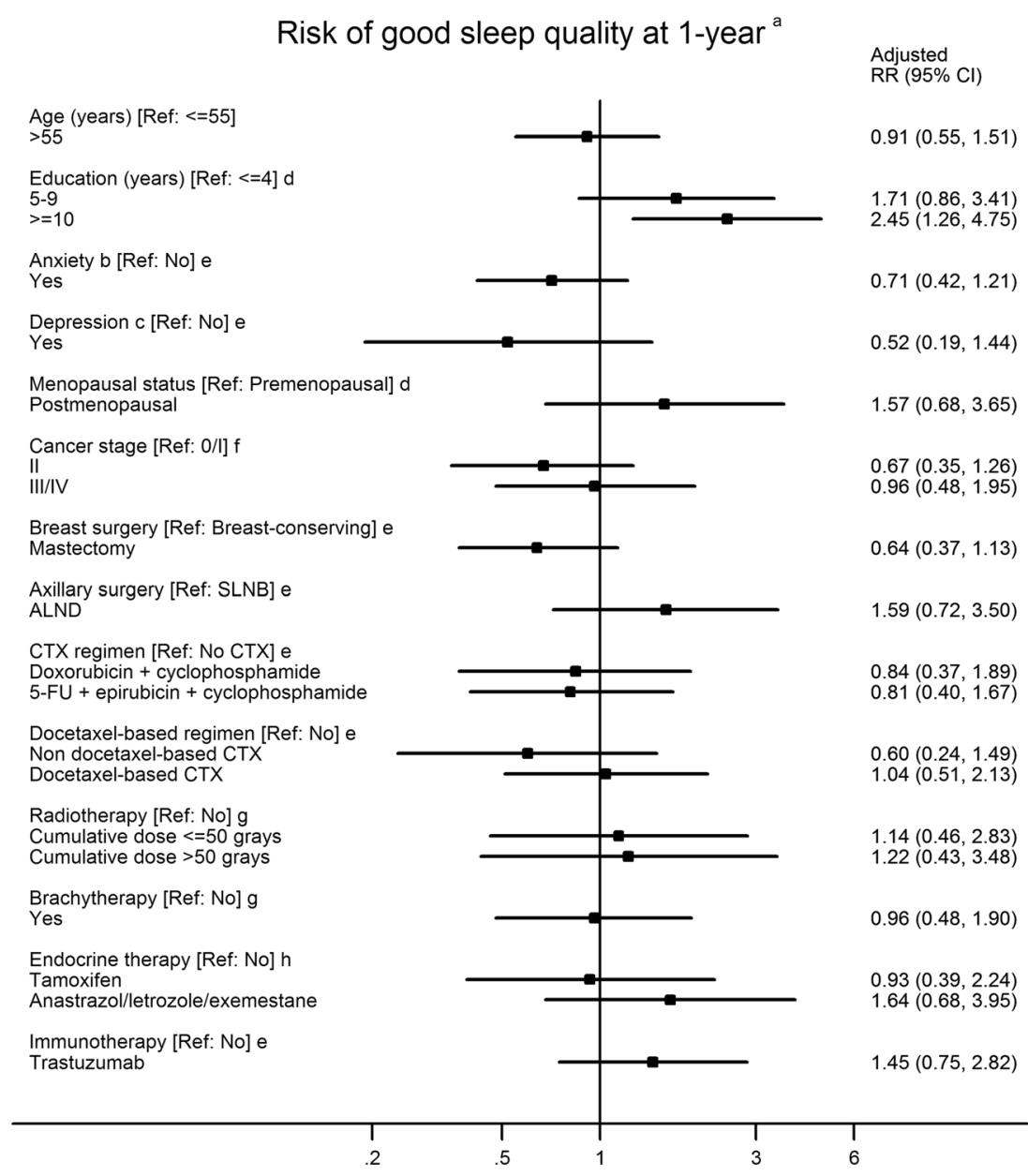

djusted

$91(0.55,1.51)$

$1.71(0.86,3.41)$

$0.71(0.42,1.21)$

$0.52(0.19,1.44)$

$1.57(0.68,3.65)$

$0.67(0.35,1.26)$
$0.96(0.48,1.95)$

$0.64(0.37,1.13)$

$1.59(0.72,3.50)$

$0.84(0.37,1.89)$

$0.60(0.24,1.49)$

$1.14(0.46,2.83)$

$0.96(0.48,1.90)$

$0.93(0.39,2.24)$

$1.45(0.75,2.82)$ polysomnography). Nevertheless, according to the recommendations for a standard research assessment of sleep or insomnia symptoms in epidemiological studies, the PSQI is one of the measures recommended to evaluate these outcomes and has been demonstrated good psychometric properties [41]. We evaluated sleep quality up to 1 year of follow-up, which limits the assessment of the long-term burden of breast cancer treatments (e.g., breast reconstruction). Nevertheless, a longer follow-up of these patients is being conducted, to further improve the information provided by this study.

In conclusion, our study shows that sleep disturbances are frequent even before cancer treatments and support its cooccurrence with other important medical conditions, such as anxiety and depression. In addition, our findings highlight the effect of different breast cancer treatments in the trajectories of sleep quality during the first year after diagnosis. These results emphasize the importance of monitoring sleep quality prior to and during treatments, and the pertinence of treatment for cooccurring disorders, as part of a strategy to improve sleep quality prior to the beginning of treatments. Additional research is needed to evaluate the existence of different patterns of sleep quality among women with breast cancer in the long term and to clarify the role of other important side effects of breast cancer treatments as mediators of the impact of treatments on sleep quality.

\section{Compliance with ethical standards}

Funding FF and ARC have received co-funded by the "Fundação para a Ciência e a Tecnologia" and the POPH/FSE Program (grant numbers SFRH/BD/92630/2013 and SFRH/BD/102181/2014, respectively). Data management activities were supported by the Chair on Pain Medicine of the Faculty of Medicine, University of Porto and by the Grünenthal Foundation - Portugal.

Conflict of interest The authors declare that they have no conflicts of interest.

Ethical approval The study was approved by the Ethics Committee of the Portuguese Institute of Oncology of Porto (Ref. CES 406/011) and by the Portuguese Data Protection Authority (Ref. 9469/2012). All participants provided written informed consent.

\section{References}

1. Ferlay J, Soerjomataram I, Ervik M et al (2013) GLOBOCAN 2012 v1.0, cancer incidence and mortality worldwide: IARC CancerBase 
No. 11. Lyon, France, International Agency for Research on Cancer http://globocan.iarc.fr, Accessed January 1, 2016

2. Amaro J, Severo M, Vilela S et al (2013) Patterns of breast cancer mortality trends in Europe. Breast 22(3):244-253

3. Allemani C, Weir HK, Carreira H et al (2015) Global surveillance of cancer survival 1995-2009: analysis of individual data for 25, 676,887 patients from 279 population-based registries in 67 countries (CONCORD-2). Lancet 385(9972):977-1010

4. Soerjomataram I, Lortet-Tieulent J, Parkin DM et al (2012) Global burden of cancer in 2008: a systematic analysis of disabilityadjusted life-years in 12 world regions. Lancet 380(9856):18401850

5. Fortner BV, Stepanski EJ, Wang SC, Kasprowicz S, Durrence HH (2002) Sleep and quality of life in breast cancer patients. J Pain Symptom Manag 24(5):471-480

6. Liu L, Fiorentino L, Rissling M et al (2013) Decreased healthrelated quality of life in women with breast cancer is associated with poor sleep. Behav Sleep Med 11(3):189-206

7. Fiorentino L, Ancoli-Israel S (2006) Insomnia and its treatment in women with breast cancer. Sleep Med Rev 10(6):419-429

8. Denieffe S, Cowman S, Gooney M (2014) Symptoms, clusters and quality of life prior to surgery for breast cancer. J Clin Nurs 23(1718):2491-2502

9. Savard J, Villa J, Ivers H, Simard S, Morin CM (2009) Prevalence, natural course, and risk factors of insomnia comorbid with cancer over a 2-month period. J Clin Oncol 27(31):5233-5239

10. Kotronoulas G, Wengstrom Y, Kearney N (2012) A critical review of women's sleep-wake patterns in the context of neo-/adjuvant chemotherapy for early-stage breast cancer. Breast 21(2):128-141

11. Knobf MT, Sun Y (2005) A longitudinal study of symptoms and self-care activities in women treated with primary radiotherapy for breast cancer. Cancer Nurs 28(3):210-218

12. Costa AR, Fontes F, Pereira S, Goncalves M, Azevedo A, Lunet N (2014) Impact of breast cancer treatments on sleep disturbances - a systematic review. Breast 23(6):697-709

13. Van Onselen C, Paul SM, Lee K et al (2013) Trajectories of sleep disturbance and daytime sleepiness in women before and after surgery for breast cancer. J Pain Symptom Manag 45(2):244-260

14. Pereira S, Fontes F, Sonin T, et al.(2014) Neurological complications of breast cancer: study protocol of a prospective cohort study. BMJ Open;4(10):e006301.

15. Freitas S, Simoes MR, Alves L, Santana I (2011) Montreal cognitive assessment (MoCA): normative study for the Portuguese population. J Clin Exp Neuropsychol 33(9):989-996

16. Edge S, Byrd DR, Compton CC, Fritz AG, Greene FL, Trotti A (2010) AJCC cancer staging manual. Springer, New York

17. De Vos FY, van Laarhoven HW, Laven JS et al (2012) Menopausal status and adjuvant hormonal therapy for breast cancer patients: a practical guideline. Crit Rev Oncol Hematol 84(2):252-260

18. Zigmond AS, Snaith RP (1983) The hospital anxiety and depression scale. Acta Psychiatr Scand 67(6):361-370

19. Pais-Ribeiro J, Silva I, Ferreira T, Martins A, Meneses R, Baltar M (2007) Validation study of a Portuguese version of the hospital anxiety and depression scale. Psychol Health Med 12(2):225-235 quiz 35-7

20. Snaith RP (2003) The hospital anxiety and depression scale. Health Qual Life Outcomes 1:29

21. Buysse DJ, Reynolds CF 3rd, Monk TH, Berman SR, Kupfer DJ (1989) The Pittsburgh sleep quality index: a new instrument for psychiatric practice and research. Psychiatry Res 28(2):193-213

22. Marques D, Gomes AA, Meiavia A, Salgueiro A, Carlos Ribeiro J, Dischler J.(2013) Reliability and initial validation of the Pittsburgh sleep quality index, European Portuguese version: a preliminary study in a sample of higher education students. Sleep Med;14(Suppl 1 ):e140.
23. Amaral MO, de Figueiredo Pereira CM, Silva Martins DI, de Serpa CR, Sakellarides CT (2013) Prevalence and risk factors for insomnia among Portuguese adolescents. Eur J Pediatr 172(10):13051311

24. Smagula SF, Stone KL, Fabio A, Cauley JA.(2015) Risk factors for sleep disturbances in older adults: evidence from prospective studies. Sleep Med Rev.

25. Papadimitriou GN, Linkowski P (2005) Sleep disturbance in anxiety disorders. Int Rev Psychiatry 17(4):229-236

26. Desai K, Mao JJ, Su I et al (2013) Prevalence and risk factors for insomnia among breast cancer patients on aromatase inhibitors. Support Care Cancer 21(1):43-51

27. Colagiuri B, Christensen S, Jensen AB, Price MA, Butow PN, Zachariae R (2011) Prevalence and predictors of sleep difficulty in a national cohort of women with primary breast cancer three to four months postsurgery. J Pain Symptom Manag 42(5):710-720

28. American Psychiatric Association (2013) Diagnostic and statistical manual of mental disorders (DSM-V). American Psychiatric Association, Washington, DC

29. Savard J, Morin CM (2001) Insomnia in the context of cancer: a review of a neglected problem. J Clin Oncol 19(3):895-908

30. Berger AM, Wielgus K, Hertzog M, Fischer P, Farr L (2010) Patterns of circadian activity rhythms and their relationships with fatigue and anxiety/depression in women treated with breast cancer adjuvant chemotherapy. Support Care Cancer 18(1):105-114

31. Ho SY, Rohan KJ, Parent J, Tager FA, McKinley PS (2015) A longitudinal study of depression, fatigue, and sleep disturbances as a symptom cluster in women with breast cancer. J Pain Symptom Manag 49(4):707-715

32. Bower JE, Ganz PA, Desmond KA, Rowland JH, Meyerowitz BE, Belin TR (2000) Fatigue in breast cancer survivors: occurrence, correlates, and impact on quality of life. J Clin Oncol 18(4):743753

33. Brown LF, Kroenke K (2009) Cancer-related fatigue and its associations with depression and anxiety: a systematic review. Psychosomatics 50(5):440-447

34. Bardwell WA, Profant J, Casden DR et al (2008) The relative importance of specific risk factors for insomnia in women treated for early-stage breast cancer. Psychooncology 17(1):9-18

35. Coles CE, Moody AM, Wilson CB, Burnet NG.(2005) Reduction of radiotherapy-induced late complications in early breast cancer: the role of intensity-modulated radiation therapy and partial breast irradiation. Part I-normal tissue complications. Clin Oncol (R Coll Radiol);17(1):16-24.

36. Bower JE, Ganz PA, Irwin MR, Kwan L, Breen EC, Cole SW (2011) Inflammation and behavioral symptoms after breast cancer treatment: do fatigue, depression, and sleep disturbance share a common underlying mechanism? J Clin Oncol 29(26):3517-3522

37. Palesh O, Zeitzer JM, Conrad A et al (2008) Vagal regulation, cortisol, and sleep disruption in women with metastatic breast cancer. J Clin Sleep Med 4(5):441-449

38. Miller AH, Ancoli-Israel S, Bower JE, Capuron L, Irwin MR (2008) Neuroendocrine-immune mechanisms of behavioral comorbidities in patients with cancer. J Clin Oncol 26(6):971-982

39. Koopman C, Nouriani B, Erickson V et al (2002) Sleep disturbances in women with metastatic breast cancer. Breast J 8(6): $362-370$

40. Savard J, Simard S, Blanchet J, Ivers H, Morin CM (2001) Prevalence, clinical characteristics, and risk factors for insomnia in the context of breast cancer. Sleep 24(5):583-590

41. Buysse DJ, Ancoli-Israel S, Edinger JD, Lichstein KL, Morin CM (2006) Recommendations for a standard research assessment of insomnia. Sleep 29(9):1155-1173 